\title{
Behavior of Electronic Interferometers in the Nonlinear Regime
}

\author{
I. Neder* and E. Ginossar \\ Department of Condensed Matter Physics, The Weizmann Institute of Science, Rehovot 76100, Israel
}

(Received 8 November 2007; published 16 May 2008)

\begin{abstract}
We investigate theoretically the behavior of the current oscillations in an electronic Mach-Zehnder interferometer (MZI) as a function of its source bias. Recently, the MZI visibility data showed an unexplained lobe pattern with a peculiar phase rigidity. Moreover, the effect did not depend on the MZI path length difference. We argue that these effects may be a new many-body manifestation of particlewave duality in quantum mechanics. When biasing the interferometer sources so much that multiple electrons are on each arm at any instant in time, quantum shot noise (a particle phenomena) must affect the interference pattern of the electrons that create it. A solution to the interaction Hamiltonian presented here shows that the interference visibility has a lobe pattern with applied bias that has a period proportional to the average path length and independent of the path length difference, together with a phase rigidity.
\end{abstract}

DOI: 10.1103/PhysRevLett.100.196806

In the past two decades electron interferometers have become a primary tool in mesoscopic physics for investigating quantum coherence of transport in semiconductors and to measure and control novel quantum effects [1-6]. These interferometers are understood so far only in the linear response regime, where a small bias is put on their sources, and the noninteracting picture is valid. In the nonlinear regime several electrons are present inside the interferometer at a given time and may form, in contrast to the optical interferometers, nontrivial many-body correlations due to Coulomb interaction. Indeed, new experiments in the nonlinear regime demonstrated that the LandauerButtiker formalism [7] seems to break down, and the interference pattern showed new peculiar behavior. Recently, an unexpected interference behavior of a Mach-Zehnder interferometer (MZI) (see Fig. 1) was reported [8,9], in which the visibility [proportional to the observed amplitude of the Aharonov-Bohm oscillations of the current] evolved in a lobe pattern with increasing the source bias, with zero visibility between the lobes, and a phase independence on the bias inside each lobe ("phase rigidity"). Similar visibility lobes were observed earlier in a twoterminal closed interferometer $[2,10]$. In the MZI case, the lobe pattern could not be explained using any noninteracting picture, mainly because the lobes were very robust against induced asymmetry between the two paths, and did not depend at all on their length difference. Explanations proposed for this effect using bosonization techniques [11-13] imposed interactions between electrons that were geometry dependent, somewhat in contrast to the robustness of the experimental observation. Moreover, these do not explain the total phase rigidity seen in the experiment [8].

Here we show that these lobes and phase rigidity are indeed very reasonably a nonperturbative result of the Coulomb interaction. We argue that in the nonlinear regime this interaction causes the extra electrons inside a two-path interferometer to form a correlated state which is rooted in their quantum behavior; as electrons behave both
PACS numbers: 85.35.Ds, 03.65.Yz, 72.70.+m, 73.23.-b

as particles and as waves, the phase of an interfering electron gets discrete "quantum kicks" according to the possible occupations of the additional electron states in the two arms. In some distinct source voltages those phase fluctuations cause the vanishing of the interference, which reappear at lower or higher voltages, leading to a lobe pattern in the visibility.

The basis for our derivation is a general principle for mesoscopic devices, which we term the Buttiker-Levinson argument (BLA): changing the chemical potential of all the sources by the same amount should not change the transmission from any source to any drain. It is generally relevant for any energy-dependant mesoscopic devices [14], and, in particular, for the MZI, being two-path interferometer formed by one-dimensional edge channels in the quantum Hall effect regime, since the interaction between the electrons inside the paths is unscreened by the gates nearby. Surprisingly, when applied to electron interferometers in the nonlinear regime, this simple argument is violated by noninteracting models [8].

A seemingly natural solution to this inconsistency is to correct the noninteracting models by taking into account a mean field approximation (MFA) $[8,10,14]$ in which the bias induces a static mean charging in each of the interfer-

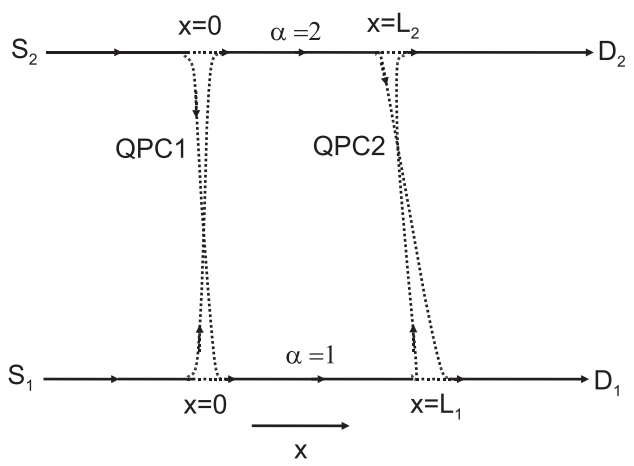

FIG. 1. Schematic of the MZI geometry, with generally asymmetric two paths. 
ometer arms which causes a phase shift due to Coulomb interaction. In comparison to experiment, however, this approximation failed since the observed lobes were independent of the two arm length difference [8]. We now further argue that this MFA correction is never valid in the MZI, since whenever it is significant, quantum fluctuations are strong and cannot be neglected. As the phase shift must restore the BLA validity, the phase shift correction in the MZI must be given by $e^{i 2 \pi\left\langle N_{1}-N_{2}\right\rangle}$ [15], where $N_{\alpha}$ is the excess number of electrons in path $\alpha$ due to the source bias. Because of the applied bias this number and the phase in each arm fluctuate binomially (quantum shot noise) with a large variance and, most importantly, with strong negative correlation between the two arms. Therefore the induced phase shift cannot be approximated simply by the average charging since $\left\langle e^{i \delta \phi(N)}\right\rangle \neq e^{i\langle\delta \phi(N)\rangle}$, as was suggested in [10]. Rather, in this situation of few electrons inside the MZI, generally $[N, \delta \phi] \neq 0$, and nonlinear interactions may cause new many-body coherent states analogous to squeezed spin states [16]. Our situation is shown below to be closely related to another nonlinear system: a biased finite 1D detector channel coupled to a MZI arm $[17,18]$.

Our starting point is the noninteracting Hamiltonian, written for spinless or spin polarized electrons in the MZI drawn in Fig. 1. It is expressed by the annihilation and creation operators $c_{k}^{\left(S_{\alpha}\right)}, c_{k}^{\left(S_{\alpha}\right) \dagger}$ for the incoming extended single-particle energy states from the two sources $S_{1,2}$,

$$
H_{0}=\sum_{\alpha=1,2} \sum_{k}\left[\epsilon(k)-\mu_{S_{\alpha}}\right] c_{k}^{\left(S_{\alpha}\right) \dagger} c_{k}^{\left(S_{\alpha}\right)} .
$$

In this model a linear relation can be established between the incoming and outgoing $c_{k}$ operators from the sources to the drains

$$
c_{k}^{\left(D_{\alpha}\right)}=\sum_{\beta=1,2} s_{k, \alpha \beta}^{(\mathrm{MZI})} c_{k}^{\left(S_{\beta}\right)},
$$

where the $s$ matrix for the particular MZI geometry can be calculated from the product $s^{(\mathrm{MZI})}=s^{(\mathrm{QPC} 2)} s^{(\delta \varphi)} s^{(\mathrm{QPC} 1)}$ with the three $s$ matrices defined as $(a=1,2)$

$$
s^{[\mathrm{QPC}(a)]}=\left(\begin{array}{cc}
i r_{a} & t_{a} \\
t_{a} & i r_{a}
\end{array}\right), \quad s^{(\delta \varphi)}=\left(\begin{array}{cc}
e^{i k L_{1}} & 0 \\
0 & e^{i k L_{2}}
\end{array}\right),
$$

where $r_{a}$ and $t_{a}$ are the reflection and transmission amplitude of the quantum point contact $\operatorname{QPC}(a)$ and $L_{1,2}$ are the two MZI path lengths. We can define the annihilation operator $c_{k}^{(\alpha)}$ of an electron moving in arm $\alpha$ of the MZI as obeying the relations

$c_{k}^{(\alpha)}=\sum_{\beta=1,2} s_{\alpha \beta}^{(\mathrm{QPC} 1)} c_{k}^{\left(S_{\beta}\right)} \quad c_{k}^{\left(D_{\alpha}\right)}=\sum_{\beta=1,2}\left[s^{(\mathrm{QPC} 2)} s^{(\delta \varphi)}\right] c_{k}^{(\beta)}$.

Considering the local nature of the interactions and of the device, we choose to express the current in the drains using the local operators after QPC2, $\psi_{\left(D_{\alpha}\right)}(x, t)=$ $\sum_{k} e^{i k x} c_{k}^{\left(D_{\alpha}\right)}(t)$. The resulting chemical potential at any drain $D_{\alpha}, \mu_{D_{\alpha}}$, is proportional to the average current to the drain, $\mu_{D_{\alpha}}=\frac{h}{e}\left\langle I_{D_{\alpha}}\right\rangle$, and since our problem is stationary in time it can be written [linearizing $\epsilon(k)$ ] using this operator $\psi_{\left(D_{\alpha}\right)}(x, t)$ at $t=0$,

$$
\mu_{D_{\alpha}}=\frac{h}{e}\left\langle I_{D_{\alpha}}\right\rangle=h v_{g}\left\langle\psi_{\left(D_{\alpha}\right)}^{\dagger}\left(L_{\alpha}, 0\right) \psi_{\left(D_{\alpha}\right)}\left(L_{\alpha}, 0\right)\right\rangle,
$$

where $v_{g}$ is the group velocity at the Fermi energy. In the noninteracting model Eq. (5) can be written as $\mu_{D_{\alpha}}=$ $\mu_{0, \alpha}+\operatorname{Re}\left(\mu_{\varphi, \alpha} e^{i \varphi_{0}}\right)$, with $\mu_{0, \alpha}$ a real parameter and $\mu_{\varphi, \alpha}$ a complex prefactor of the phase $\left(\varphi_{0}=k_{F} \Delta L\right)$ dependant term. The second term is proportional to $\int_{\mu_{S_{1}}}^{\mu_{S_{2}}} \cos \left[\varphi_{0}+\right.$ $\left.\frac{\Delta L}{\hbar v_{g}}\left(\epsilon-E_{F}\right)\right] d \epsilon=\left[2 \hbar v_{g} \sin \left(\frac{\Delta L \Delta \mu}{\hbar v_{g}}\right)\right] \cos \left[\varphi_{0}+\frac{\Delta L}{\hbar v_{g}}(\bar{\mu}-\right.$ $\left.E_{F}\right)$ ], with $E_{F}$ the Fermi energy at zero bias. It violates BLA through the explicit dependence on the average source bias $\bar{\mu}-E_{F}$.

Since, as stated above, a MFA correction is inadequate due to the quantum fluctuations, we are forced to return to the Hamiltonian, and introduce additional nonlinear Coulomb terms (whose range we assume here to be longer then the MZI arms) to take care of the BLA:

$$
H=H_{0}+\sum_{\alpha=1,2} \frac{e^{2}}{2 C_{\alpha}} N_{\alpha}^{2}
$$

where $N_{\alpha}(t)=\int_{0}^{L_{\alpha}} \rho_{\alpha}(x, t) d x$ is the number operator which counts the electrons which are added to the MZI path $\alpha$ region at time $t, \rho_{\alpha}(x, t)=\psi_{\alpha}^{\dagger}(x, t) \psi_{\alpha}(x, t)$, and $C_{\alpha}=\frac{e^{2} L_{\alpha}}{h v_{g}}$ is the electric capacitance of path $\alpha$, induced by the dispersion in $H_{0}$ and Pauli principle. Hence, the added terms in the Hamiltonian do not have additional free parameters.

The role of the additional term is to locally raise the bottom of the conduction band (the electrostatic potential) in path $\alpha$ according to its overall local charging. The effect is exactly such that when a full beam between energies $E_{F}$ and $E_{F}+\Delta \mu$ enters the path, the last fully occupied state remains with the same momentum $k_{F}$, with a new energy $\bar{\mu}=E_{F}+\Delta \mu$, which restores BLA validity. We should note that in addition to this dynamic effect, the new terms also add electron-hole excitations above the Fermi sea, which we ignore [19].

We shall solve now approximately the equation of motion for the interacting case. Keeping the definition in Eq. (5) and expecting the same functional dependence of $\mu_{D_{\alpha}}$ on $\varphi_{0}$ as in the noninteracting case, we want to show that $\mu_{\varphi, \alpha}$ develops a lobe structure behavior and phase rigidity with an energy scale independent of $\Delta L$. The equation of motion for $\psi_{\alpha}(x, t)$ reads 


$$
\begin{aligned}
\frac{\partial \psi_{\alpha}(x, t)}{\partial t}= & \frac{1}{i \hbar}\left[\psi_{\alpha}(x), H\right] \\
= & v_{g} \frac{\partial \psi_{\alpha}}{\partial x}-i \frac{\pi v_{g}}{L_{\alpha}}\left[\psi_{\alpha}(x, t) N_{\alpha}\right. \\
& \left.+N_{\alpha} \psi_{\alpha}(x, t)\right] \Theta(x) \Theta\left(L_{\alpha}-x\right),
\end{aligned}
$$

where $\Theta(x)$ is the Heaviside function. We use the linear relations in Eq. (4) to define the operators $\psi_{\alpha}(x, t)$ at $x<0$ and at $x>L_{\alpha}$,

$$
\begin{aligned}
& \psi_{\left(D_{\alpha}\right)}(x, t)=\sum_{\beta=1,2} s_{\alpha \beta}^{(\mathrm{QPC} 2)} \psi_{(\beta)}(x, t), \quad x>L_{\alpha}, \\
& \psi_{(\alpha)}(x, t)=\sum_{\beta=1,2} s_{\alpha \beta}^{(\mathrm{QPCl})} \psi_{\left(S_{\beta}\right)}(x, t), \quad x<0,
\end{aligned}
$$

where $\psi_{\left(S_{\alpha}\right)}(x, t)=\sum_{k} e^{i k x} c_{k}^{\left(S_{\alpha}\right)}(t)$. Having the noninteracting solution $\psi_{\alpha}(x, t)=\psi_{\alpha}^{(0)}\left(x-v_{g} t\right)$ at $x<0$ as a boundary condition, Eq. (7) has a unique formal solution:

$$
\psi_{\alpha}(x, t)= \begin{cases}\psi_{\alpha}^{(0)}\left(x-v_{g} t\right) & x<0 \\ \mathcal{T}\left[U_{\alpha}(t)\right] \psi_{\alpha}^{(0)}\left(x-v_{g} t\right) \mathcal{T}^{-1}\left[U_{\alpha}(t)\right] & 0<x<L_{\alpha} \\ \psi_{\alpha}\left[L_{\alpha}, t-\left(x-L_{\alpha}\right) / v_{g}\right] & L_{\alpha}<x,\end{cases}
$$

where $\mathcal{T}$ is the time-ordering operator, and we define

$$
U_{\alpha}(t)=e^{-\left(i \pi v_{g} / L_{\alpha}\right) \int_{t-x / v_{g}}^{t} d t^{\prime} N_{\alpha}\left(t^{\prime}\right)} .
$$

Equation (9) is difficult to evaluate analytically, due to the fact that $U_{\alpha}(t)$ depends on $\psi_{\alpha}(x, t)$. However, one can obtain some general properties of the highly correlated quantum state at the drains, using the following derivation. First we approximate $U_{\alpha}(t)$ by replacing $\rho_{\alpha}(x, t)$ in $N_{\alpha}(t)$ with the noninteracting density $\rho_{\alpha}^{0}\left(x-v_{g} t\right)$. One should note that this is a very crude approximation as electrons really repel each other and change each other's group velocity considerably; hence, the true solution might be different in its fine details. However, note that unlike the MFA, this approximation maintains the non-Gaussian behavior of the shot noise as well as the negative correlation of the shot noise between the two paths. Next, due to the fact that $N^{0}(t)$ are bosonic, $\left[N_{\alpha}^{0}(t), N_{\alpha}^{0}\left(t^{\prime}\right)\right]$ is a $C$ number, so the $T$ ordering can be eliminated by repeatedly using Baker-Hausdorff formula, such that the phases originating from the $T$ and the $T^{-1}$ ordering cancel each other. Then, we take $t=0$ and rearrange the operator in the exponent $U_{\alpha}=U_{\alpha}(t=0)=e^{-i \phi_{\alpha}}$ as a weighted integral over the density operator $\phi_{\alpha}=\int_{-\infty}^{\infty} w_{\alpha}(x) \rho_{\alpha}^{0}(x) d x$. Straightforward calculation shows $w_{\alpha}(x)$ to be a triangle

$$
w_{\alpha}(x)= \begin{cases}\pi\left(1-\left|\frac{x-L_{\alpha}}{L_{\alpha}}\right|\right) & \left|x-L_{\alpha}\right|<L_{\alpha} \\ 0 & \left|x-L_{\alpha}\right|>L_{\alpha} .\end{cases}
$$

An additional approximation is to restrict the energy excitations in the operators $\rho_{\alpha}^{0}(x)$ and therefore also in the operator $\phi_{\alpha}$,

$$
\phi_{\alpha} \approx \tilde{\phi}_{\alpha}=\sum_{k, k^{\prime}=k_{F}}^{k_{F}+\Delta \mu / \hbar v_{g}} w_{\alpha, k-k^{\prime}} c_{\alpha, k}^{\dagger} c_{\alpha, k^{\prime}},
$$

where $w_{\alpha, k-k^{\prime}}$ is the Fourier transform of $w_{\alpha}(x)$, and $\tilde{O}$ denote an operator restricted to the energies $\left[E_{F}, E_{F}+\right.$ $\Delta \mu]$. This approximation is very intuitive as it takes into account only dephasing due to excess shot noise, disregarding virtual transitions to energies above $E_{F}+\Delta \mu$. It was proven very useful in explaining the unique experimental results of dephasing by shot noise [17]. With these approximations, the prefactor of the phase-dependant part of Eq. (5) reads

$$
\mu_{\varphi}=h v_{g}\left\langle\Psi_{0}\left|\tilde{U}_{1}^{\dagger} \tilde{U}_{2} \tilde{\psi}_{1}^{0 \dagger}\left(L_{1}\right) \tilde{\psi}_{2}^{0}\left(L_{2}\right) \tilde{U}_{1}^{\dagger} \tilde{U}_{2}\right| \Psi_{0}\right\rangle,
$$

where $\left|\Psi_{0}\right\rangle=\left(\prod_{k=k_{F}}^{k_{F}+\Delta \mu / \hbar v_{g}} c_{k}^{\left(S_{1}\right) \dagger}\right)\left|g s\left(\mu_{S_{1,2}}=E_{F}\right)\right\rangle$. Equation (13) can be evaluated by noting that $\tilde{\phi}_{1}$ and $\tilde{\phi}_{2}$ commute, so one can introduce a basis of a complete set of eigenstates of the two operators $\left\{\left|\varphi_{1}, \varphi_{2}\right\rangle\right\}$. Note that the single-particle matrix $w_{\alpha, k, k^{\prime}}$, restricted to the voltage window $\left[E_{F}, E_{F}+\Delta \mu\right]$, has a discrete set of nonvanishing eigenvalues, corresponding to localized electron states near the finite influence region of $w(x)$ [Eq. (11)]. The eigenstate $\left|\varphi_{\alpha}\right\rangle$ of the operator $\tilde{\phi}_{\alpha}$ is a specific choice of occupations of those single-particle states, the value $\varphi_{\alpha}$ being the sum of the eigenvalues of the occupied states. With two insertions of such a full set, Eq. (13) now reads

$$
\begin{aligned}
\mu_{\varphi}= & h v_{g} \sum_{\varphi_{1}, \varphi_{2}, \varphi_{1}^{\prime}, \varphi_{2}^{\prime}}\left\langle\Psi_{0} \mid \varphi_{1}, \varphi_{2}\right\rangle\left\langle\varphi_{1}\left|\tilde{\psi}_{1}^{0 \dagger}\left(L_{1}, 0\right)\right| \varphi_{1}^{\prime}\right\rangle \\
& \times\left\langle\varphi_{2}\left|\tilde{\psi}_{2}^{0}\left(L_{2}, 0\right)\right| \varphi_{2}^{\prime}\right\rangle\left\langle\varphi_{1}^{\prime}, \varphi_{2}^{\prime} \mid \Psi_{0}\right\rangle e^{i\left(\varphi_{1}-\varphi_{2}+\varphi_{1}^{\prime}-\varphi_{2}^{\prime}\right)} .
\end{aligned}
$$

This expression differs from the noninteracting one only by
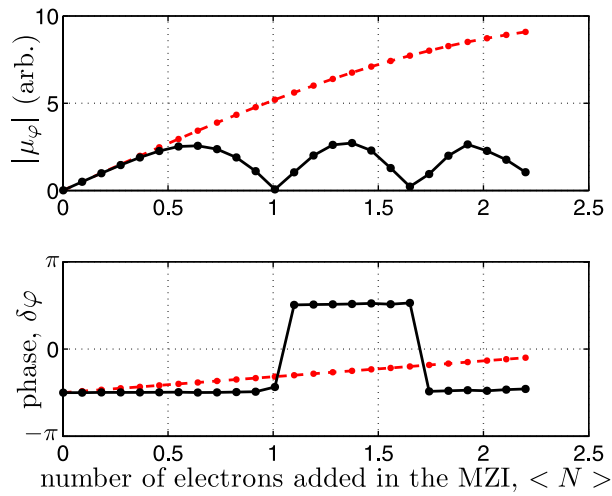

FIG. 2 (color online). The amplitude and phase shift of the interference oscillations in the chemical potential of the MZI drain, as a function of $\langle N\rangle=\bar{L} \Delta \mu / h v_{g}$ in the noninteracting model [gray (red) curves] and in presence of the interaction [Eq. (13), black curves], for $\langle\Delta N\rangle /\langle N\rangle=\Delta L / \bar{L}=0.2$. The lines between the calculated points are a guide to the eye. Note that we plotted here the total current visibility, which is more suitable for analyzing the lobes then the differential visibility [9]. 


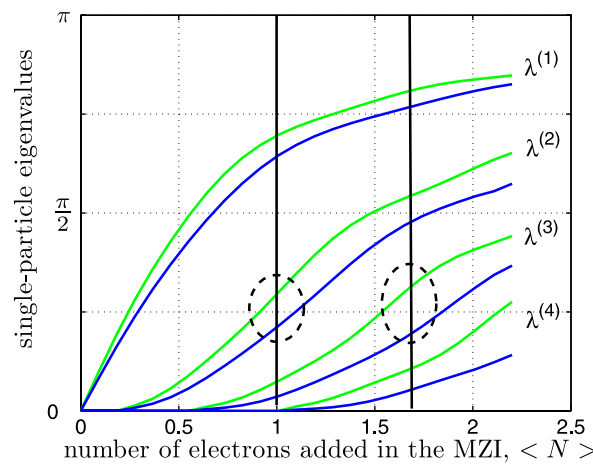

FIG. 3 (color online). The pairs of the single-particle eigenvalues of the phase operators $w_{1}$ and $w_{2}$ of the two paths [dark gray (blue) and light gray (green) lines], as a function of $\langle N\rangle$, for $\langle\Delta N\rangle /\langle N\rangle=0.2$. Black lines mark the zero visibility in Fig. 2. Note that at every such zero, there is a pair of eigenvalues whose sum is $\pi / 2$.

the exponent at the end of the right-hand side of the equation. Equation (14) can be evaluated numerically, through diagonalization of the matrices $w_{\alpha, k, k^{\prime}}$. Figure 2 shows the result for the visibility, as a function of $\langle N\rangle=$ $\left\langle N_{1}+N_{2}\right\rangle=\frac{\bar{L}}{h v_{g}} \Delta \mu$, at $\frac{\langle\Delta N\rangle}{\langle N\rangle}=\frac{\Delta L}{\bar{L}}=0.2$, and $\left|t_{1}\right|^{2}=$ $\left|t_{2}\right|^{2}=0.5$. The prediction of the noninteracting model is also plotted for comparison.

One can clearly see that while in the noninteracting model the visibility and phase are slowly varying (because the scale on which they change is proportional to $\Delta L^{-1}$ ), in the interacting model a lobe pattern appears in the visibility, with a stick-slip behavior of the phase. The lobe's evolution is much faster, apparently proportional to $\langle N\rangle^{-1}$ and hence to $\bar{L}^{-1}$, in agreement with the experimental results. Figure 3 shows the evolution of the eigenvalues of the single-particle matrices $w_{1}$ and $w_{2}$ as a function of $\langle N\rangle$.

At small bias there are two nonzero eigenvalues, one for each of the phase operators. When put in the exponents of Eq. (14), they exactly cancel the kinetic phase induced from $H_{0}$, resulting in phase rigidity. The first zero of the visibility occurs at $\langle N\rangle=1$. As seen from Fig. 3, at this value the sum of the second pair of single-particle eigenvalues of the two arms is exactly $\lambda_{1}^{(2)}+\lambda_{2}^{(2)}=\pi / 2$. This can be understood by assuming that the operators $\tilde{\psi}_{1}^{0 \dagger}\left(L_{1}, 0\right)$ and $\tilde{\psi}_{2}^{0}\left(L_{2}, 0\right)$ in Eq. (14) act effectively only on the first single-particle state [the numeric calculation indeed shows that the contribution of the other electron transitions in Eq. (14) is negligible]. However, the phase of these two operators is modulated by $\varphi^{(1)}-\varphi^{(2)}+\varphi^{(1)}-$ $\varphi^{\prime(2)}$, which contains also the occupation of the second eigenstates. As the second electron can occupy the lower arm or the upper arm, a zero visibility happens when the difference in the added phase between these two options becomes $\pi$, so they coherently cancel each other. Quite generally, the visibility goes to zero whenever a sum of a pair of a single-particle eigenvalues of the two paths reaches $\pi / 2$.
Compared with the experiment, the theory still has shortcomings. The first is that the visibility lobes do not have the overall decaying envelope. Moreover, differentiating the curve in Fig. 2 near $\langle N\rangle=1$ leads to a phasedependant differential response $d \mu_{D_{\alpha}, \varphi} / d \mu_{S_{1}}$ which is twice that of the linear response - a differential visibility of $200 \%$. These might be a nonphysical result of our approximations, which will be absent in the exact solution of Eq. (7).

In conclusion, in this Letter we investigated the effect of quantum shot noise inside electronic interferometers on the interference visibility in the nonlinear regime. We established that an interaction Hamiltonian such as in Eq. (6) must always replace the noninteracting one, which quite generally leads to lobe pattern of the visibility with increasing source bias. In the future it would be desirable to apply the theory developed here to other mesoscopic devices such as two-terminal $A B$ interferometers [2,10], MZI working in the fractional quantum Hall regime, or twoparticle interferometers [5,6].

We wish to thank A. Stern, Y. Levinson, and M. Heiblum for long and very useful discussions.

*izhar.neder@weizmann.ac.il

[1] G. Timp, A. M. Chang, J.E. Cunningham, T. Y. Chang, P. Mankiewich, R. Behringer, and R.E. Howard, Phys. Rev. Lett. 58, 2814 (1987).

[2] A. Yacoby, R. Schuster, and M. Heiblum, Phys. Rev. B 53, 9583 (1996).

[3] E. Buks, R. Schuster, M. Heiblum, D. Mahalu, and V. Umanski, Nature (London) 391, 871 (1998).

[4] Y. Ji, Y. Chung, D. Shprinzak, M. Heiblum, D. Mahalu, and H. Shtrikman, Nature (London) 422, 415 (2003).

[5] P. Samuelsson, E. V. Sukhorukov, and M. Büttiker, Phys. Rev. Lett. 91, 157002 (2003).

[6] I. Neder, N. Ofek, Y. Chung, M. Heiblum, D. Mahalu, and V. Umansky, Nature (London) 448, 333 (2007).

[7] M. Buttiker, IBM J. Res. Dev. 32, 63 (1988).

[8] I. Neder, M. Heiblum, Y. Levinson, D. Mahalu, and V. Umansky, Phys. Rev. Lett. 96, 016804 (2006).

[9] P. Roulleau, F. Portier, D. C. Glattli, P. Roche, A. Cavanna, G. Faini, U. Gennser, and D. Mailly, Phys. Rev. B 76, 161309 (2007).

[10] W. van der Wiel et al., Phys. Rev. B 67, 033307 (2003).

[11] E. V. Sukhorukov and V. V. Cheianov, Phys. Rev. Lett. 99, 156801 (2007).

[12] J. T. Chalker, Y. Gefen, and M. Y. Veillette, Phys. Rev. B 76, 085320 (2007).

[13] I. P. Levkivskyi and E. V. Sukhorukov, arXiv:0801.2338.

[14] T. Christen and M. Büttiker, Europhys. Lett. 35, 523 (1996).

[15] J. Friedel, Philos. Mag. 43, 153 (1952).

[16] M. Kitagawa and M. Ueda, Phys. Rev. Lett. 67, 1852 (1991).

[17] I. Neder, F. Marquardt, M. Heiblum, D. Mahalu, and V. Umansky, Nature Phys. 3, 534 (2007).

[18] I. Neder and F. Marquardt, New J. Phys. 9, 112 (2007).

[19] F. Marquardt, Europhys. Lett. 72, 788 (2005). 